\title{
Diseño y manufactura de ortesis dinámica para mano mediante el modelado por deposición fundida (MDF)
}

\section{Design and manufacture of dynamic orthotics for hand using molten deposition modeling (MDM)}

GUANDULAY-ALCÁZAR, Miguel $\dagger^{*}$, LEDESMA-JAIME, Reynaldo, FERRER-ALMARAZ, Miguel y RAMOS LÁZARO, Gabriela

Universidad Tecnológica del Suroeste de Guanajuato, Carretera Valle-Huanímaro km 1.2, Valle de Santiago, Gto.

ID $1^{\text {er }}$ Autor: Miguel, Guandulay-Alcázar /ORC ID: 0000-0002-8831-9547, Researcher ID Thomson: S-6750-2018, CVU CONACYT ID: 443671

ID $1^{\text {er }}$ Coautor: Reynaldo, Ledesma-Jaime / ORC ID: 0000-0001-9484-3305, Researcher ID Thomson: S-6786-2018, CVU CONACYT ID: 165239

ID $2^{\text {do }}$ Coautor: Miguel, Ferrer-Almaraz / ORC ID: 0000-0003-4913-4010, Researcher ID Thomson: S-6969-2018, CVU CONACYT ID: 342076

ID $3^{\text {er }}$ Coautor: Gabriela, Ramos-Lázaro / ORC ID: 0000-0002-8827-3948, Researcher ID Thomson: S-7730-2018, CVU CONACYT ID: 439753

DOI: $10.35429 / J O H S .2019 .20 .6 .23 .31$

Recibido Julio 15, 2019; Aceptado Septiembre 20, 2019

\begin{abstract}
Resumen
Actualmente una gran cantidad de lesiones en la mano se presentan en la población Mexicana, principalmente ocasionados por accidentes en el trabajo y en actividades deportivas. Para ayudar a la población a tener acceso a una rehabilitación braquiopalmar, en este trabajo se propuso desarrollar un prototipo rehabilitador "ortesis dinámica para mano" para atender las principales patologías que afectan a nuestra sociedad (fracturas, luxaciones, esguinces, tendinitis y sx del túnel del carpo). El diseño fue analizado en elemento finito para la validación del mismo teniendo un factor de seguridad de 2.6 y una tensión máxima de $12.84 \mathrm{MPa}$ por debajo del límite elástico del ABS, la fabricación se realizó mediante una impresora 3D con el filamento ABS. Esta ortesis tiene un aditamento extensor de dedos compuesta por hamacas, una para cada dedo. Debido a su diseño universal, puede ser utilizado para ambas manos solo intercambiando de posición la extensión del dedo pulgar.
\end{abstract}

Ortesis, Modelado, deposición fundida

\begin{abstract}
Currently a large number of hand injuries occur in the Mexican population, mainly caused by accidents at work and in sports activities. To help the population have access to a brachiopalmar rehabilitation, In this work it was proposed to develop a rehabilitative prototype "dynamic hand orthosis" to address the main pathologies that affect our society (fractures, dislocations, sprains, tendonitis and sx of the carpal tunnel). The design was analyzed in a finite element for its validation, having a safety factor of 2.6 and a maximum tension of $12.84 \mathrm{MPa}$ below the ABS elastic limit, Manufacturing was done using a 3D printer with the ABS filament. This orthosis has an extensor finger attachment composed of hammocks, one for each finger. Due to its universal design, it can be used for both hands only by exchanging the extension of the thumb.
\end{abstract}

Orthosis, Modeling, molten deposition

Citación: GUANDULAY-ALCÁZAR, Miguel, LEDESMA-JAIME, Reynaldo, FERRER-ALMARAZ, Miguel y RAMOS LÁZARO, Gabriela. Diseño y manufactura de ortesis dinámica para mano mediante el modelado por deposición fundida (MDF). Revista de Ciencias de la Salud. 2019. 6-20: 23-31.

*Correspondencia al Autor (Correo Electrónico: maguandulay@utsoe.edu.mx)

$\dagger$ Investigador contribuyendo como primer Autor 


\section{Introducción}

La mano del hombre es una excelente herramienta, capaz de ejecutar innumerables acciones gracias a su función esencial: la prensión. Está dotada de una gran riqueza funcional que le procura una abundancia de posibilidades en las posiciones, los movimientos y las acciones, Deval J. (2008).

Desde el punto de vista fisiológico, la mano representa la extremidad efectora del miembro superior. Sin embargo, esta no es sólo un órgano de ejecución, es también un receptor sensorial extremadamente sensible y preciso cuya información es indispensable para retroalimentar su propia acción, Moore KL. (2002). La ortesis de miembro superior son dispositivos que adaptados externamente sobre cualquier región anatómica de la extremidad superior modifican sus características estructurales o funcionales, con el fin de mantener, mejorar o restaurar la función alterada de la citada extremidad o de alguno de sus segmentos, consejo interterritorial del sistema nacional de salud (2001).

Estas férulas se pueden clasificar en, estáticas (posicionales): Dispositivos que no permiten movimiento, pero mantienen una determinada postura. Se utilizan como un soporte rígido en fracturas, condiciones inflamatorias de tendones y partes blandas e injurias nerviosas (ver Figura 1), y en dinámicas (funcionales o cinéticas): En contraste con las ortesis estáticas, estos dispositivos permiten o facilitan el movimiento. Este tipo de ortéticos es utilizado principalmente para asistir al movimiento de músculos debilitados (ver Figura 2).
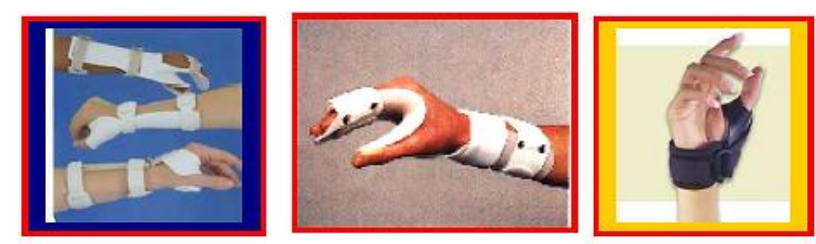

Figura 1 Ortesis estáticas

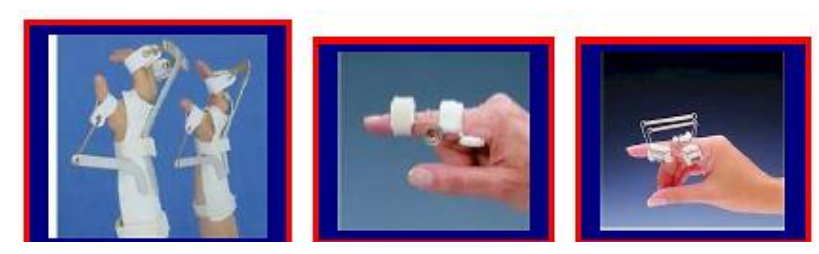

Figura 2 Ortesis dinámicas
Los dispositivos estáticos previenen el movimiento y, por tanto, permiten el reposo de la parte inmovilizada. Debe inmovilizarse "únicamente" la articulación cuyo movimiento intenta impedirse y, en consecuencia, tiene que moldearse anatómicamente según los contornos de la parte incluida y no debe ejercer presiones indebidas sobre prominencias óseas o en áreas por donde discurren paquetes vasculonerviosos. Los dispositivos dinámicos permiten, guían, limitan o resisten movimientos específicos $\mathrm{y}$ previenen movimientos determinados. Es conveniente que se conozcan con exactitud los movimientos que deben evitarse, así como el movimiento que hay que asistir o resistir. Igualmente debe definirse el límite del movimiento deseado. Pueden utilizar fuentes internas de fuerza (acción muscular) o fuentes externas (bandas de caucho, resortes, barras de tensión o fuentes eléctricas o electrónicas), Arce, G. (2005).

\section{Justificación}

La rehabilitación de la mano del ser humano requiere de la ayuda de aparatos eficientes para llegar a obtener un porcentaje adecuado de recuperación en la movilidad, así mismo es de vital importancia evitar las mayores incidencias de secuelas para que sean reincorporados a su vida cotidiana o laboral. Para ello se propone fabricar un prototipo en impresión 3D que ayude a una rehabilitación adecuada.

\section{Objetivo General}

Diseñar y construir una ortesis para mano mediante el modelado por deposición fundida para atender las principales patologías que afectan a nuestra sociedad.

\section{Marco Teórico}

\section{Patologías que afectan a la biomecánica de la mano}

\section{Fractura}

Una fractura se define como la interrupción de la continuidad ósea y/o cartilaginosa. Habitualmente se producen como consecuencia de un traumatismo único de intensidad superior a la que el hueso sano puede soportar, (ver Figura 3). Clínicamente las fracturas pueden ocasionar dolor, deformidad e impotencia funcional. 
El diagnóstico se confirma mediante radiografía simple en al menos dos proyecciones (generalmente antero posterior y lateral). Una fractura es conminuta cuando en el foco se aprecian varios fragmentos, a continuación, en la figura 3 se muestra los nombres de cada fractura dependiendo de la zona, Gutiérrez, D. F. (1945).

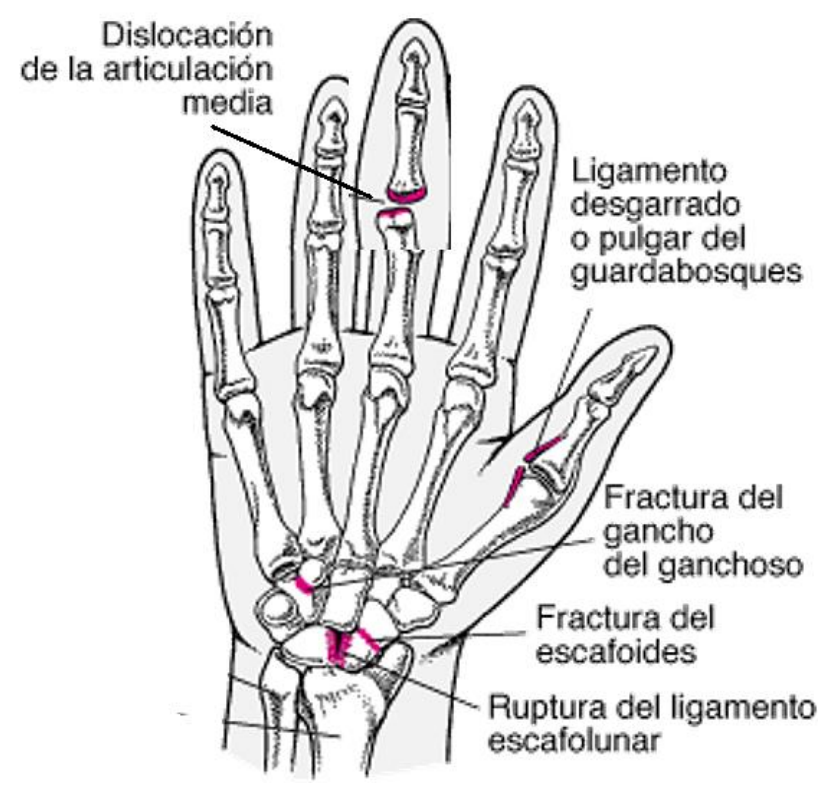

Figura 3 Tipos de fracturas en la mano

\section{Luxación}

Una luxación es una lesión que consiste en la separación de las dos partes de una articulación (Figura 4). Se produce como consecuencia de la aplicación violenta de una fuerza física. Las luxaciones suelen provocar dolores intensos e inflamación en las zonas afectadas. Las partes luxadas tienden también a deformarse y son muy difíciles de mover una vez resultan afectadas. A diferencia de lo que ocurre con los esguinces, los huesos separados no regresan en esta ocasión a la posición inicial. En caso de luxación, se recomienda inmovilizar la articulación dañada, aplicar hielo sobre ella y mantenerla en reposo absoluto. En ningún caso tratar de recolocar ni aplicar masajes. Las luxaciones más habituales son las de hombro, codo, cadera y dedos, producto la mayoría de las veces de caídas fortuitas. El principal riesgo en todos los casos es que un hueso desplazado comprima o desgarre los vasos sanguíneos y/o los nervios que pasan cerca, Hoppenfeld, S. (2004).

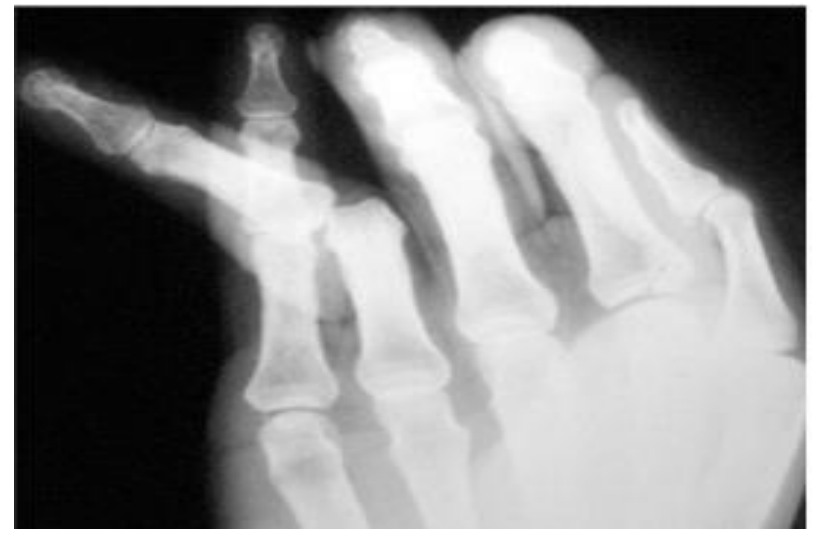

Figura 4 Luxación en la mano

\section{Esguince}

Estiramiento o rasgadura de los ligamentos, el tejido fibroso que conecta los huesos y las articulaciones. Un esguince de muñeca Figura 5, puede estar relacionado con una lesión por practicar deportes o con un traumatismo. Los síntomas son la hinchazón, el dolor, la aparición de moretones y la sensibilidad de la muñeca. El reposo, las compresas de hielo, los analgésicos y la elevación de la muñeca pueden ser de ayuda. Un médico puede inmovilizar la articulación con una férula, en la figura 5 se muestra un ejemplo de un esguince en la muñeca, Silberman, F. S. (2010).

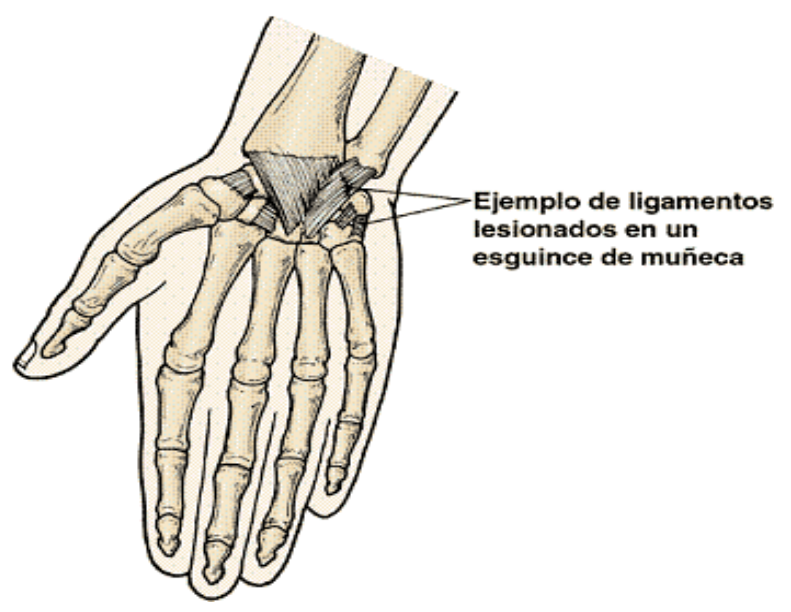

Figura 5 Esguince en la muñeca

\section{Sx de túnel de carpo}

Es la neuropatía por compresión más frecuente de todo el organismo. Su cuadro clínico clásico se da en mujeres de edad media avanzada y está dominado por la aparición de parestesias en primer, segundo, tercer y mitad radial del cuarto dedo, inicialmente de predominio nocturno y casi siempre en la mano dominante (si bien en muchos casos es bilateral). 
La maniobra de Phalen se realiza manteniendo una flexión máxima de la muñeca durante un minuto; es positiva si se reproducen las parestesias en el territorio del mediano. Las parestesias se reproducen también mediante percusión sobre la zona comprimida (signo de Tinnel). Como métodos diagnósticos se emplean la radiología simple y la electromiografía. La aparición de atrofia en la eminencia ténar condiciona mal pronóstico. El tratamiento conservador está justificado sólo cuando la causa es transitoria; en caso contrario debe procederse a la sección quirúrgica del ligamento volar transverso del carpo, en la Figura 6 se muestra el descompresión del nervio mediano en el túnel carpiano, Silberman., F. S. (2010).

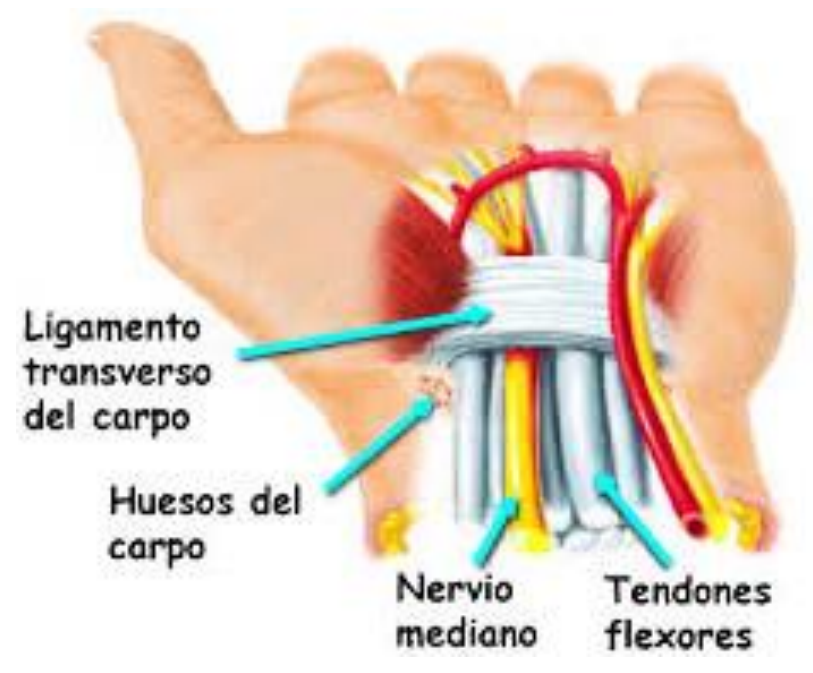

Figura 6 Descompresión del nervio mediano en el túnel carpiano

\section{Ortesis dinámica para mano}

Férula activa extensora de articulaciones metacarpofalángicas y aditamento extensor de dedo/s

Consta de un apoyo antebraquial y tres apoyos en termoplástico semirrígido almohadillado, Figura 7: Uno en la cara dorsal sobre metacarpianos y dos en la cara palmar, uno a nivel de las falanges proximales y otro a nivel de los metacarpianos. Estos dos soportes están unidos al soporte dorsal con dos alambres de acero, que discurren por los bordes medial y lateral del segundo y quinto dedos. A su vez, el soporte dorsal está unido, con dos alambres de acero forrados, a una abrazadera que abarca la muñeca y que se ajusta con una cincha autoadhesiva. Los alambres laterales configuran el sistema de tracción elástica o resorte de este dispositivo.

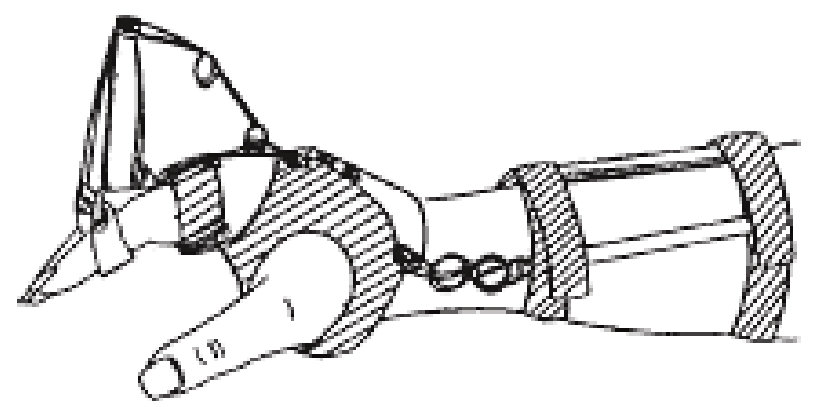

Figura 7 Férula activa extensora de articulaciones

Este dispositivo tiene un aditamento extensor de dedos compuesto por hamacas, una para cada dedo, con apoyo palmar en falanges distales o segundas falanges (dependiendo que se quiera conseguir la extensión de todo el dedo o sólo de la articulación interfalángica proximal) que se unen directamente, mediante alambres acerados curvados, con el soporte dorsal metacarpofalángico.

Existen modelos que pueden llevar bloqueo de la articulación de la muñeca y/o aditamento de pulgar. Consejo interterritorial del sistema nacional de salud (2001).

\section{Férula activa flexora de articulacione metacarpofalángicas y aditamento flexor de dedo/s}

Férula dinámica construida de alambre acerado y de termoplástico semirrígido almohadillado, que consta de un apoyo antebraquial y de un apoyo dorsal sobre las falanges proximales, un apoyo palmar sobre articulaciones metacarpofalángicas y una correa sobre la cara dorsal de la mano. El apoyo palmar se une al dorsal mediante dos alambres acerados en espiral situados medial y lateralmente y el palmar se une a la correa dorsal mediante un alambre acerado angulado, Figura 8.

Este prototipo tiene un aditamento flexor para dedo/s, forrado, de apoyo dorsal en la segunda interfalange o sobre articulaciones interfalángicas distales. Este soporte está unido mediante dos gomas elásticas que parten del borde interno y externo, con el apoyo dorsal sobre articulaciones metacarpofalángicas proximales.

Existen modelos que pueden llevar bloqueo de la articulación de la muñeca y/o aditamento de pulgar. Consejo interterritorial del sistema nacional de salud (2001). 


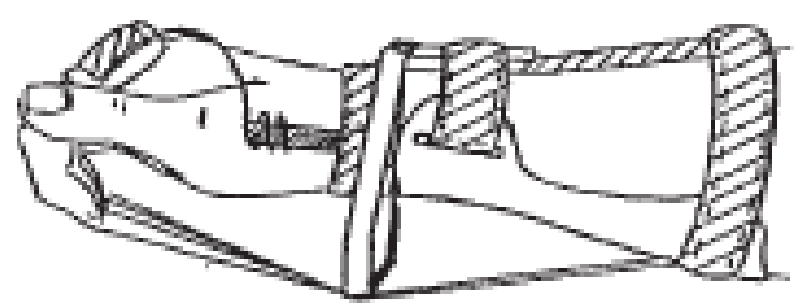

Figura 8 Férula activa flexora de articulaciones

\section{Férula activa flexora de articulaciones} metacarpofalángicas

La férula dinámica construida de alambre acerado y de termoplástico semirrígido almohadillado, consta de un apoyo antebraquial $\mathrm{y}$ de un apoyo dorsal sobre las falanges proximales, un apoyo palmar sobre articulaciones metacarpofalángicas y una correa sobre la cara dorsal de la mano.

El apoyo palmar se une al dorsal mediante dos alambres acerados en espiral situados medial y lateralmente y el palmar se une a la correa dorsal mediante un alambre acerado angulado, Figura 9. Esta ortesis tiene un aditamento extensor para dedo/s, compuesto por una/s hamaca/s, que se apoya sobre la articulación interfalángica distal del dedo o dedos en los que se quiere mantener la extensión, que se unen mediante unos alambres acerados curvados, forrados con el soporte dorsal. Existen modelos que pueden llevar bloqueo de la articulación de la muñeca y/o aditamento de pulgar. Consejo interterritorial del sistema nacional de salud (2001).

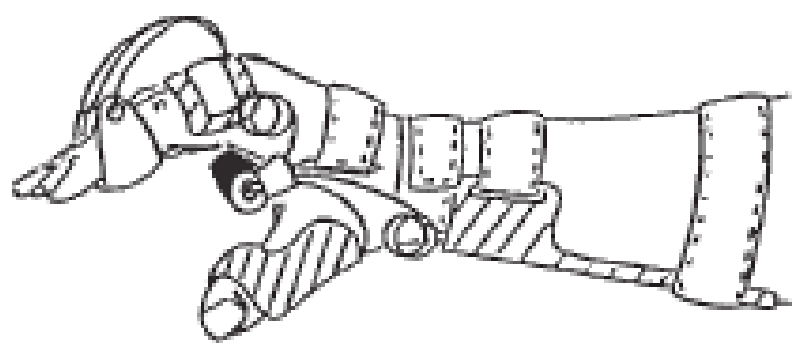

Figura 9 Férula activa flexora de articulaciones y aditamento extensor de dedo/s

\section{Desarrollo}

\section{Condiciones de diseño}

Para el desarrollo del prototipo, se debe tomar en cuenta los siguientes aspectos para la factibilidad el diseño:
- Ergonómico.

- Resistente.

- Compatible para ambas manos.

- De uso fácil.

- Portátil.

- Bajo costo de producción.

\section{Metodología del diseño}

La metodología utilizada toma como referencia el modelo de French para el proceso de diseño de la férula dinámica para mano, el cual está basado en:

- Análisis del problema.

- Diseño conceptual.

- Dar forma a los esquemas.

- Desarrollo de detalles.

En la Figura 10 se muestra el diseño conceptual, en la Figura 11 se da forma al esquema, y en la Figura 12 se hace el desarrollo de detalles, ésta es la que se toma como diseño final.

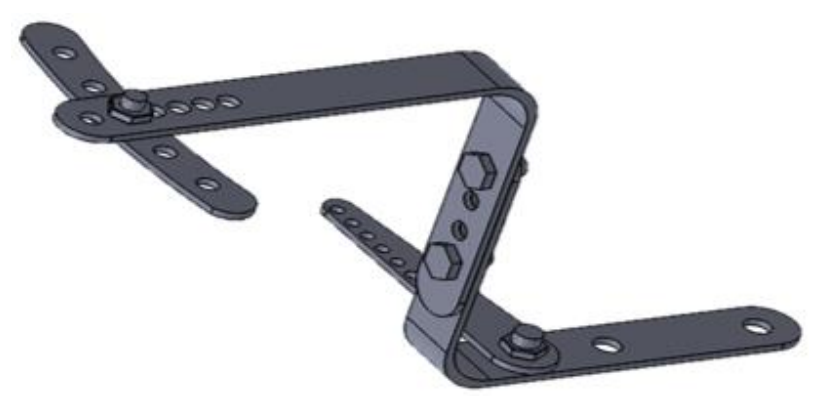

Figura 10 Diseño conceptual

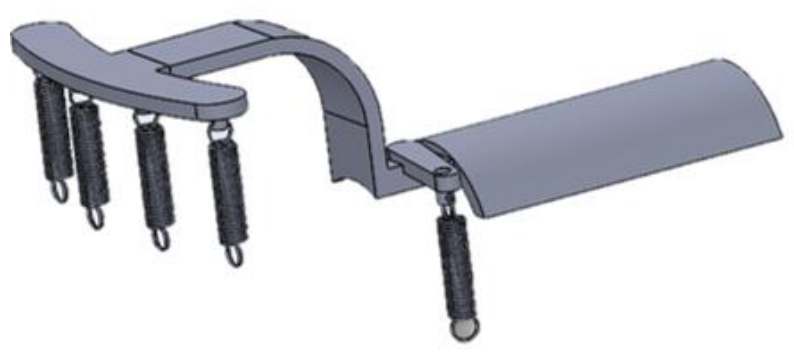

Figura 11 Dar forma al esquema

\section{Diseño final de la ortesis}

Después de considerar los puntos del modelo de French, se obtiene el diseño final del prototipo, Figura 12. 


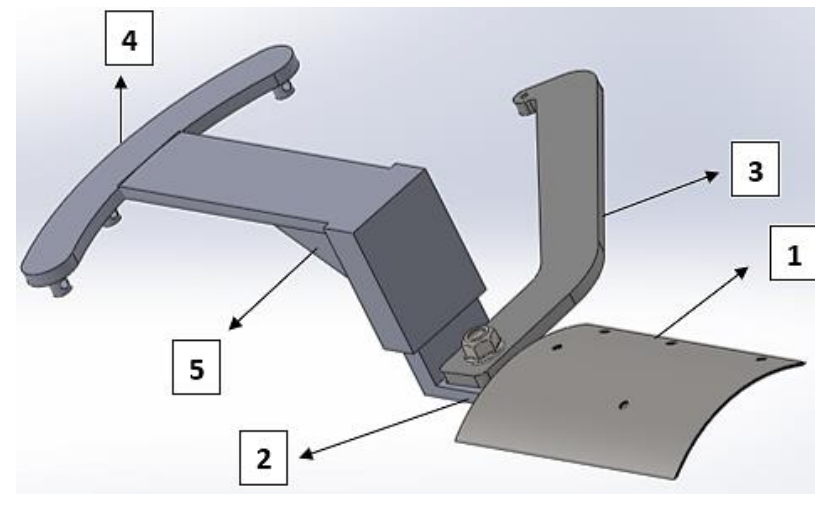

Figura 12 Diseño de prototipo final.

En la tabla 1 se muestra los elementos que conforman el modelo de la ortesis.

\begin{tabular}{|r|l|}
\hline \multicolumn{1}{|c|}{ No. } & \multicolumn{1}{|c|}{ Elemento } \\
\hline 1 & Sujetador del brazo \\
\hline 2 & Base de sujeción \\
\hline 3 & Extensión para el dedo pulgar \\
\hline 4 & $\begin{array}{l}\text { Base de sujeción para resortes (para dedos } \\
\text { meñique, anular, medio e índice) }\end{array}$ \\
\hline 5 & $\begin{array}{l}\text { Apoyo para disminuir concentración de } \\
\text { esfuerzos }\end{array}$ \\
\hline
\end{tabular}

Tabla 1 Elementos del modelo final

\section{Análisis del modelo de la ortesis}

Para la implementación de un análisis de elemento finito al dispositivo, se calculó una fuerza aproximada de $36.46 \mathrm{~N}$ equivalente a 3.7 $\mathrm{Kg}$, esta fuerza es la que sería ejercida por los cuatro dedos (meñique, anular, medio e índice) al elemento 4 de la Figura 12 al momento de la rehabilitación. Este dato se obtuvo midiendo la deformación aproximada que cada dedo ejercía sobre el resorte multiplicándolo por la constante de éste. Para llevar a cabo este análisis es indispensable colocar una sujeción de geometría fija en la parte inferior de la base de sujeción, en la Figura 13 se muestra el área de carga y la sujeción.

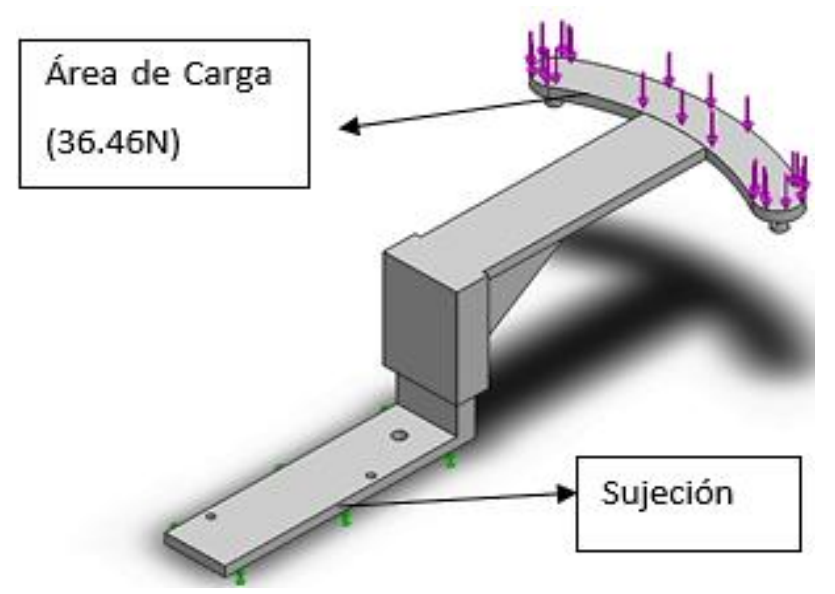

Figura 13 Área de carga y sujeción

\section{Resultado del análisis de tensiones}

De acuerdo a los resultados del análisis de tención del prototipo, Figura 14, los valores de tensión máxima no superan a los de fluencia del material, debido a que la zona de tención mínima es de 0.0004514 Pa., ubicado en el sujetador del brazo y una tensión máxima de $12.84 \mathrm{MPa}$., en la base de resortes para dedos, tomando en cuenta que el límite elástico del material $\mathrm{ABS}$ es de $39 \mathrm{MPa}$.

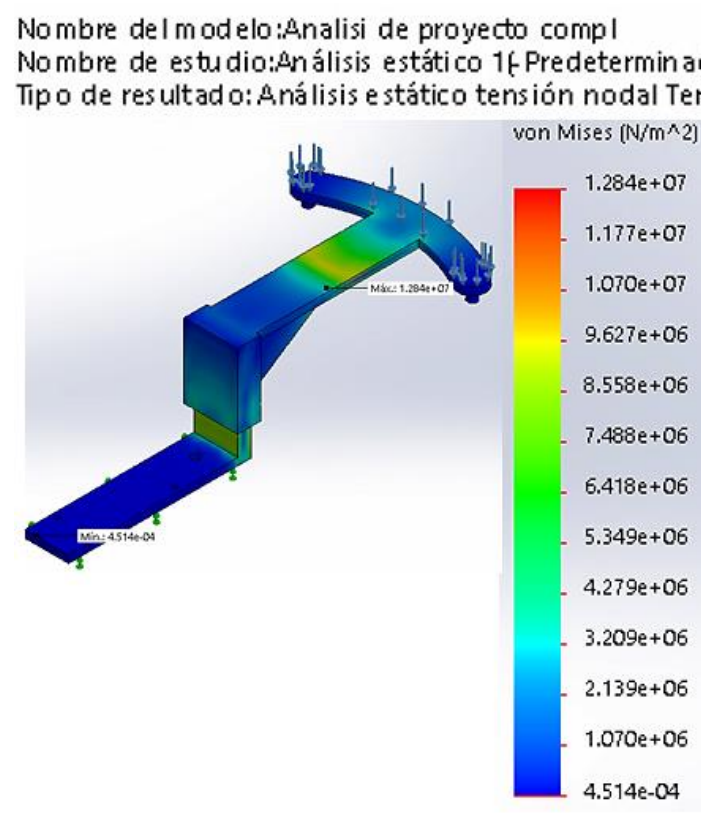

Figura 14 Análisis de tensiones

\section{Resultados del análisis de deformación}

Las deformaciones máximas Figura 15, están ubicadas en la parte frontal de la base de sujetadores de resortes para dedos, siendo de un desplazamiento de $9.92 \mathrm{~mm}$, siendo éste, aceptable dentro del funcionamiento de la ortesis. 
Nombre del modelo:Analisi de proyecto compl

Nombre de estudio:Análisis estático 1(-Predetermin ado-)

Tipo de resultado: Des plaza miento estŕtico Desplazamientos 1 Es cal a de defom ación: 1

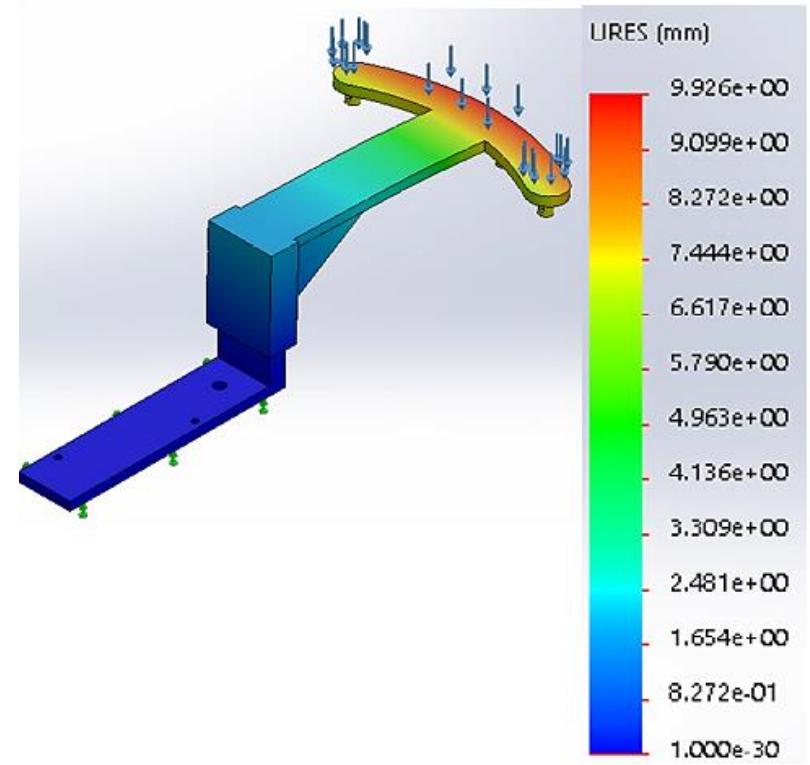

Figura 15 Análisis de deformaciones

\section{Resultado del análisis del factor de seguridad}

Los resultados obtenidos del análisis del factor de seguridad, Figura 16, arrojaron un valor mínimo de 2.6, ubicado en la parte superior de la base de resortes para dedos, siendo este un dispositivo seguro para su funcionamiento en la rehabilitación.

Nombre deImodelo:Analisi de proyecto compl

No mbre de estudio:Análisis estático 1(-Predetermin ado-)

Tipo de resultad o: Factor de seguridad Factor de seguridad2 Crite rio: Butomátion

Distribu dón de factor de se guridad: FDS mín $=2.6$

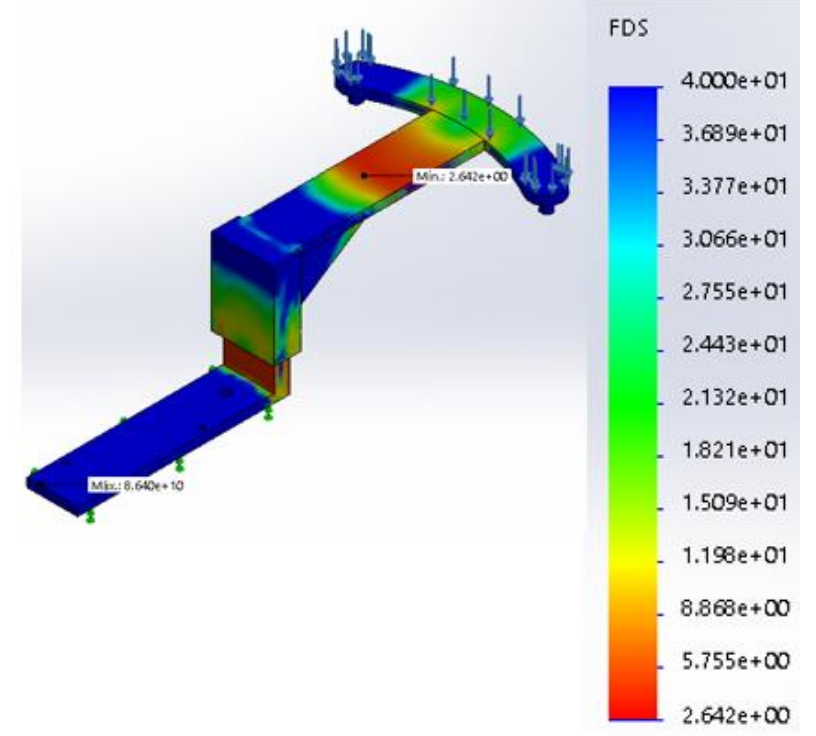

Figura 16 Análisis del factor de seguridad

\section{Modelado por deposición fundida (MDF) de la ortesis dinámica}

En el MDF del prototipo rehabilitador se utilizó una impresora 3D con filamento ABS y una lámina de $0.53 \mathrm{~mm}$ de espesor con dimensiones de $140 \mathrm{~mm}$ X $125 \mathrm{~mm}$, ésta última se utilizó para sujetar el dispositivo con el brazo como se aprecia en la Figura 12, elemento 1. Para la elaboración de cada una de las piezas, es necesario importar el diseño en CAD al programa Repetier-Host V1.6.2 de la impresora, al importar este diseño el programa genera todos los códigos para que la impresora modele la pieza.

\section{Modelado de la base de sujeción}

En el modelado de la base de sujeción Figura 17, se utilizó $13488 \mathrm{~mm}$ de filamento de ABS, el tiempo de impresión fue de 1 hora 29 minutos.

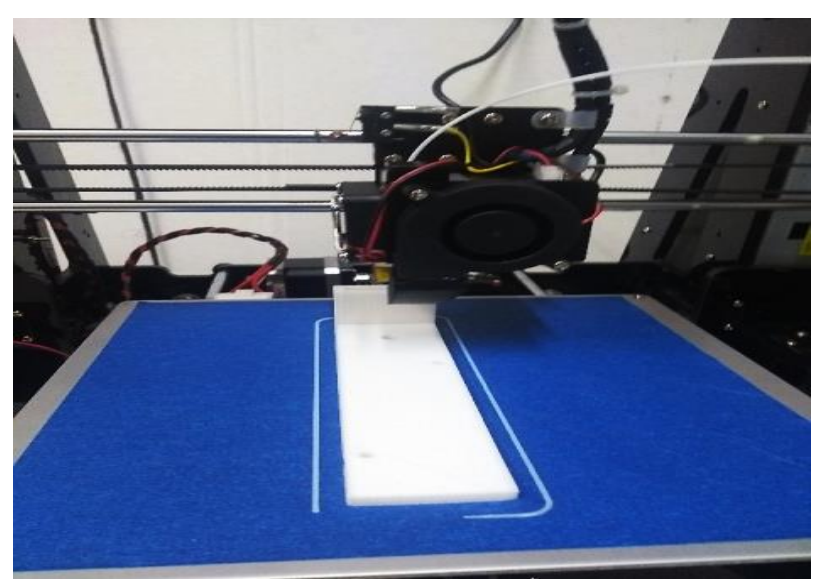

Figura 17 Base de sujeción

\section{Modelado de la extensión para el dedo pulgar}

Para la elaboración de la extensión del dedo pulgar se requirió de $10296 \mathrm{~mm}$ de filamento ABS Figura 18, el tiempo estimado de impresión fue de 1 hora 8 minutos.

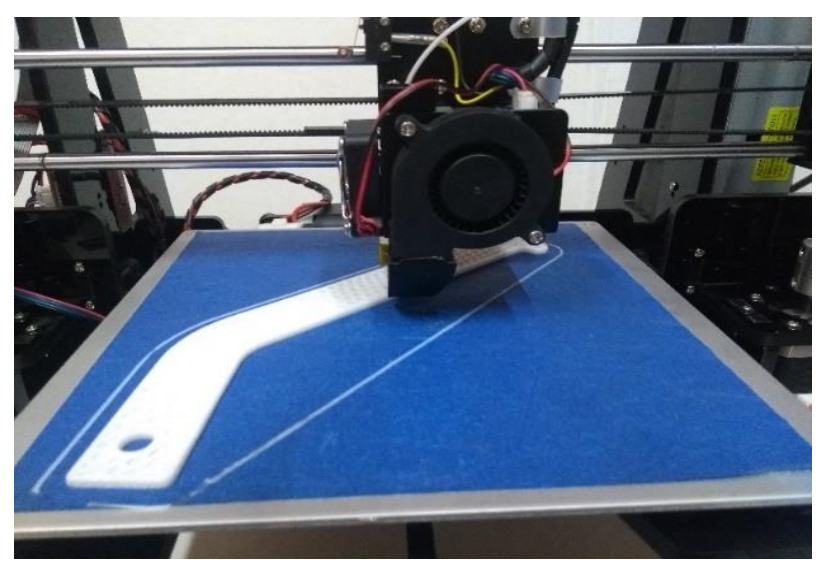

Figura 18 Extensión para el dedo pulgar

GUANDULAY-ALCÁZAR, Miguel, LEDESMA-JAIME, Reynaldo, FERRER-ALMARAZ, Miguel y RAMOS LÁZARO, Gabriela. Diseño y manufactura de ortesis dinámica para mano mediante el modelado por deposición fundida (MDF). Revista de Ciencias de la Salud. 2019 


\section{Modelado de la base de sujeción para resortes}

En la elaboración de la base de resortes para dedos (meñique, anular, medio e índice) Figura 19 se requirió de $31446 \mathrm{~mm}$ de filamento, con un tiempo de impresión de 3 hora 9 minutos y 51 segundos.

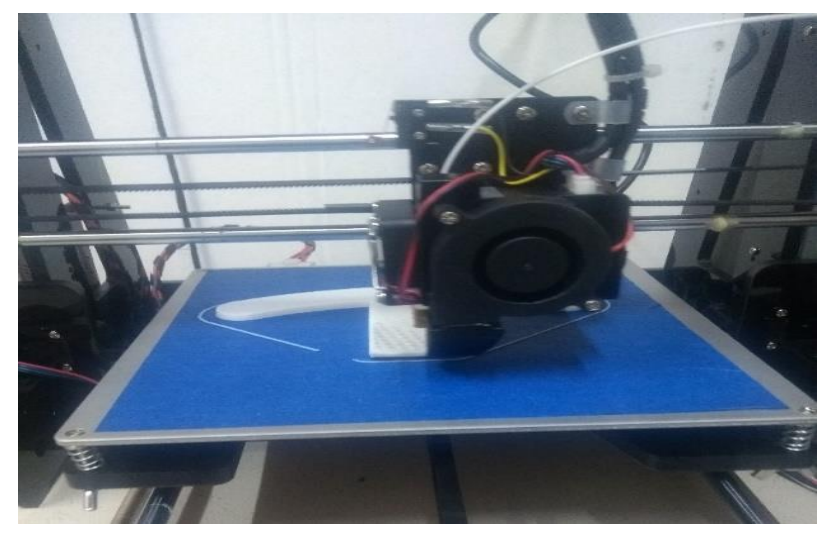

Figura 19 Base de sujeción para resosrtes

\section{Filete para reducción de esfuerzos}

Para el modelado del filete para reducción de esfuerzos Figura 20, se requirió de $6935 \mathrm{~mm}$ de filamento, con un tiempo de impresión de 39 minutos.

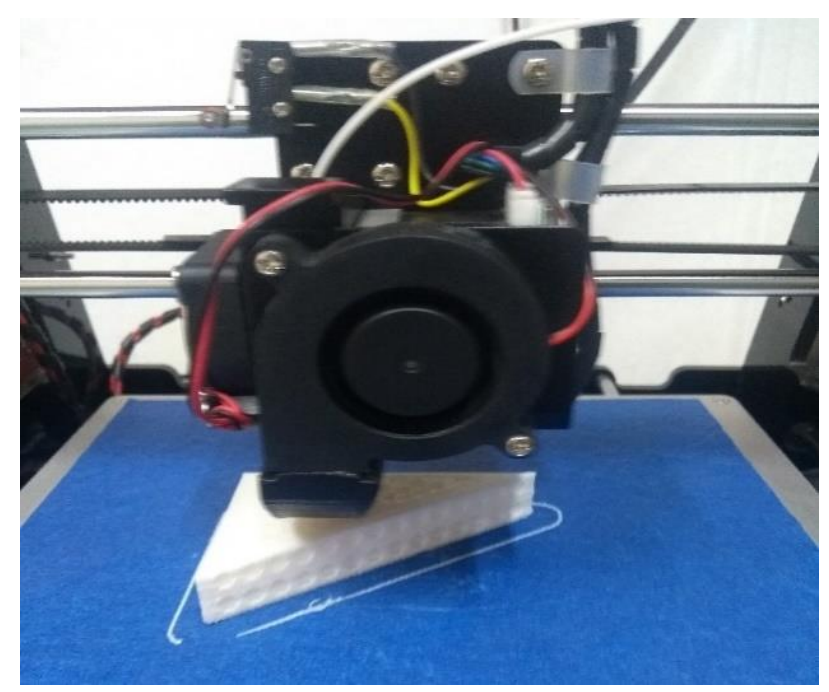

Figura 20 Filete para reducción de esfuerzos

\section{Ortesis dinámica para mano}

Como resultado de este proyecto se fabricó un dispositivo rehabilitador Figura 21, es ideal para la rehabilitación de las diferentes patologías que afectan a la mano y/o muñeca, por su diseño, es funcional para todas las personas en general, además es compatible para ambas manos.

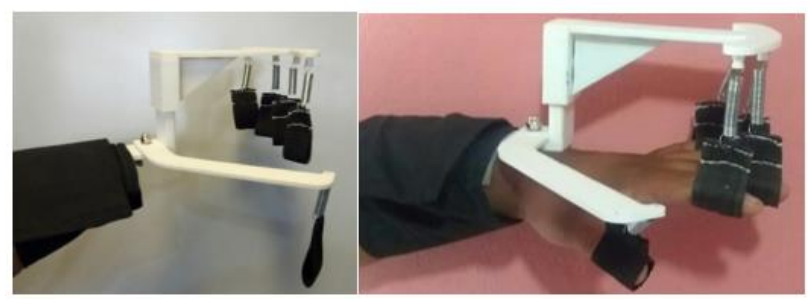

a)

b)

Figura 21 a) Ortesis dinámica para mano, b) Ortesis en posición de uso

\section{Conclusiones}

Es de vital importancia tener una rehabilitación adecuada ya que de ésta depende que nuestro sistema osteomuscular tenga una adecuada recuperación para así obtener una mejoría tanto funcional como motora y así mismo evitar la mayoría de incidencias de secuelas. Se están elaborando 10 dispositivos más para verificar la funcionalidad, éstos se harán entrega a pacientes que tengan algunas de las siguientes patologías (fractura, luxación, esguince, sx del túnel del carpo) y/o que no tengan el recurso para tener una rehabilitación, la entrega del dispositivo se hará con el apoyo de especialistas en el área, esto para llevar una valoración especializada de los pacientes con el fin de obtener ideas y aportaciones que ayuden a seguir mejorando este dispositivo.

\section{Referencias}

Andrioly, V., Nelson, M. J., \& Prieto Palencia, C. A. Diseño de una órtesis activa de rodillapara la primera etapa de la rehabilitación.

Arce, G. (2005). Ortesis de miembros superiores. Clasificación, funciones, prototipos, características, Indicaciones [Internet]. Lima, Perú: Medicina de rehabilitación.

Consejo interterritorial del sistema nacional de salud (2001). Guía descriptiva de ortoprótesis (tomo II). Recuperado .https://www.mscbs.gob.es/profesionales/presta cionesSanitarias/CarteraDeServicios/Contenido CS/6PrestacionOrtoprotesica/docs/GuiaDescrip tivaOrtoprotesisTomo2.pdf

Deval J. (2008). El desarrollo humano. España: Editorial Siglo XXI;. p. 143-45.

Ferrari-Portafaix, C. (2019). Férulas de reposo del pie del adulto: desde la ortesis de serie hasta el moldeado a medida. EMC-Podología, 21(2), $1-6$.

GUANDULAY-ALCÁZAR, Miguel, LEDESMA-JAIME, Reynaldo, FERRER-ALMARAZ, Miguel y RAMOS LÁZARO, Gabriela. Diseño y manufactura de ortesis dinámica para mano mediante el modelado por deposición fundida (MDF). Revista de Ciencias de la Salud. 2019 
Gudiño-Lau, J., Rosales, I., Charre, S., Alcalá, J., Duran, M., \& Vélez-Díaz, D. (2019). Diseño y Construcción de un Exoesqueleto para Rehabilitación. XIKUA Boletín Científico de la Escuela Superior de Tlahuelilpan, 7(13), 1-10.

Gutiérrez, D. F. (1945). Ánatomia humana. En D. F. Gutiérrez, Ánatomia humana (pág. 501). México, DF.: PORRÚA.

Hoppenfeld, S. (2004). Fractura, tratamiento y rehabilitación. En S. Hoppenfeld, Fractura, tratamiento y rehabilitación. (pág. 602). Phyladelphia: Marbán libros, S.L.

Moore KL. (2002). Fundamentos de Anatomía con orientación clínica. 4ta. Ed. Editorial Médica Panamericana: p. 777, 795.

Rojas, L., \& Eslit, R. (2019). Abordaje fisioterapéutico de anteversión femoral en niños.

Silberman., F. S. (2010). Ortopedia y traumatología. En F. S. Silberman., Ortopedia y traumatología (pág. 455). Buenos Aires: PANAMERICANA.

Undurraga, S., Saxton, F., \& Gammon, B. (2019). Prótesis total de muñeca, una alternativa para la artrosis radiocarpiana. Contacto Científico, 9(1). 